\title{
Movilidad de estudiantes a Europa: un proceso colonial inacabado
}

\author{
Student movility to Europe: an unfinished colonial process
}

\author{
Edorta Camino Esturo • edorta.camino@ehu.eus \\ UNIVERSIDAD DEL PAÍS VASCO (UPV/EHU)
}

Recibido: 28/02/2017

Aceptado:30/05/2017

\section{Resumen}

El presente artículo analiza y compara la movilidad de estudiantes internacionales que realizan su formación universitaria en Europa, tomando como modelos a cuatro países seleccionados por compartir un bagaje colonial similar: España, Francia, Portugal y Reino Unido. A través del tratamiento estadístico y cuantitativo de la base de datos de la UNESCO, se realiza un análisis de la información basado en el cálculo del Índice de Elegibilidad y en la relación entre el idioma de origen y el idioma de destino para señalar la presencia del factor histórico y cultural en las características de los desplazamientos de estudiantes extranjeros a Europa. Estos resultados ofrecen una clasificación ordenada en cuatro escenarios o modelos de movilidad yuxtapuestos: por un lado, según el ranking de movilidad de cada país observado, se encontrarían divididos en las categorías de centro y periferia, y por otro lado, según el origen colonial de la movilidad, estarían agrupados en domésticos y globalizados.

Palabras clave: Movilidad internacional de estudiantes, Colonialismo, Ecología de Saberes, Índice de Elegibilidad, Universidad.

\section{Abstract}

This article analyzes and compares the mobility of international students who complete their university education in Europe, taking as models four countries identified by a shared colonial baggage: Spain, France, Portugal and the United Kingdom. Through the statistical and quantitative treatment of the UNESCO database, an analysis of the information based on the calculation of the Eligibility Index and the relationship of the mother tongue with the language of the place of destination is performed to indicate the presence of the factor Historical and cultural aspects of the movement of foreign students to Europe. These results offer a classification what is ordered in four stage or mobility models: according to the ranking of each country, there are two categories: center and periphery; according to the colonial origin of mobility: domestic and globalized.

Keywords: International Student Mobility, Colonialism, Ecology of Knowledge, Eligibility Index, University. 


\section{INTRODUCCIÓN}

La educación universitaria es cada vez más global. Las relaciones que se desarrollan en las diferentes universidades de todos los continentes propician diversos programas de movilidad estudiantil, tipo Erasmus. Las experiencias vitales y académicas llevadas a cabo en esos países producen unos nexos y unas hibridaciones culturales no exentas de una cierta aculturación y homogeneidad basadas en el discurso hegemónico del modelo de educación occidental y su epistemología dominante. Autores como Boaventura de Sousa Santos observan una universidad hegemónica, colonialista y no democrática en estos procesos globales que reducen las posibilidades de emancipación y diversidad epistemológica (Santos, 2007 y 2010). Según lo reflejado en las dinámicas de la educación superior, se ha producido un auge espectacular de las movilidades de estudiantes que persiguen una formación cualificada a nivel universitario, en una creciente competencia por conseguir los mayores rankings de productividad científica. Esta competición establece unas categorías intermedias entre ganadores y perdedores, con la acumulación de los medios tecnológicos y del conocimiento en manos de unos pocos países desarrollados (Altbach y Knight, 2006) que ven cómo aumentan las movilidades internacionales de manera unidireccional y a su favor.

En este orden global, el objetivo del estudio es describir, analizar y comparar la movilidad internacional de estudiantes que se produce desde cualquier país del mundo a cuatro países europeos, seleccionados en base a su pasado colonial: España, Francia, Portugal y Reino Unido.

Desde el pasado siglo, el aumento de la movilidad por motivos de estudios superiores universitarios ha provocado un creciente interés académico debido a su correspondencia con los amplios procesos de globalización y las relaciones internacionales, convirtiéndose en una temática de pesquisa cada vez más importante y viendo multiplicadas sus publicaciones a nivel nacional e internacional (Altbach y Knight, 2006; Luchilo, 2006; Altbach, 2008; Valle y Garrido, 2009; Haug, 2010; Gürüz, 2011; Costa y Faria, 2012; Bermúdez, 2015; Parra, 2016). El presente artículo constituye un aporte a esta línea de investigación que, desde un enfoque postcolonial, trata de comprender el sentido cultural y colonial de las movilidades mediante un procedimiento esencialmente cuantitativo.

Utilizando los datos estadísticos del portal de la UNESCO, el tratamiento de las bases de datos a través de Excel ha posibilitado analizar la cantidad de movilidad de estudiantes y correlacionarlos según el origen de cada país o territorio con los países europeos seleccionados. Algunos de estos países y territorios todavía mantienen relaciones culturales, lingüísticas y (ex)coloniales, entre otras, con las (ex)metrópolis, lo cual requiere la elaboración de una tabla en la que quede reflejada esta circunstancia. Estas movilidades de países excoloniales, coloniales y no coloniales se suman en forma de aportaciones (inputs) al ecosistema de saberes del país de acogida o anfitrión, produciendo una interrelación entre los conocimientos científicos y hegemónicos europeos con los saberes que provienen de otros lugares. Esta cuantificación basada en el origen de las movilidades y, por tanto, de los co- 
nocimientos que las acompañan dibuja una morfología diferenciada para cada uno de estos ecosistemas de saberes.

Finalmente, el cálculo del Índice de Elegibilidad ha permitido comparar la predilección de los estudiantes que se desplazan a cada país de acogida y establecer unas tipologías en función del tamaño del indicador. Este Índice de Elegibilidad permite obtener resultados que puedan mostrar las desigualdades de elección entre los países europeos y establecer unas categorías para ordenar la relevancia cuantitativa de las movilidades a Europa.

\section{UN ENFOQUE SOBRE LA MOVILIDAD INTERNACIONAL DE ESTUDIANTES: CONCEPTOS Y TEORÍA}

El Instituto de Estadística de la UNESCO (UIS) publicó un informe en el que se observaban las tendencias mundiales en la movilidad de estudiantes y exponía que, en los primeros años del siglo xxi, este fenómeno había aumentado de manera "espectacular» a nivel global (UNESCO, 2006: 34). Lo cual no significa que sólo se produzcan movilidades en ciertas regiones mundiales más favorecidas económicamente, sino que se dan en «todas las regiones del mundo» (Bermúdez, 2015: 99) o, al menos, en regiones donde existe una mayor pobreza económica. El informe comunicaba que, sólo desde el año 1999 al 2004, aumentó un $41 \%$ la movilidad de estudiantes, incrementándose desde 1,75 a 2,5 millones de estudiantes de nivel terciario que estudiaron fuera de su país de origen (UNESCO, 2006: 34). Esta tendencia ha ido aumentando progresivamente hasta la actualidad, pudiéndose apreciar, de modo comparativo, los datos ofrecidos por los diferentes organismos internacionales como son la UNESCO, Eurostat o el Banco Mundial.

La movilidad internacional se concibe como «un flujo específico de la migración calificada" (Bermúdez, 2015: 96), caracterizada por dos perfiles de estudiantes: por un lado «aquellos estudiantes que durante el desarrollo de su formación educativa alternan estudio con trabajo" y, por otro lado, "aquellos que, terminando su ciclo de escolaridad, permanecen en la sociedad de destino y se insertan laboralmente cambiando su condición migratoria» (Bermúdez, 2015: 98). Por su parte, el Instituto de Estadística de la UNESCO define al estudiante internacional como aquella persona que «sale de su país o territorio de origen y se traslada a otro país o territorio con el fin de estudiar» (UNESCO, 2006: 33) y se ajusta a las condiciones siguientes de:

—Ciudadanía: «si no son ciudadanos del país anfitrión donde estudian».

— Residencia permanente: «si no son residentes permanentes del país anfitrión donde estudian».

—Educación previa: «si obtuvieron la calificación de admisión a su nivel actual de estudio en otro país. Por regla general, para los estudiantes matriculados en programas de nivel terciario, la educación previa es el nivel más alto de la educación secundaria». 
En cuanto al origen geográfico de los estudiantes internacionales, el UIS señala que «los datos nacionales pueden dar a los responsables de formular políticas una perspectiva clara sobre los estudiantes matriculados en los sistemas educativos nacionales de nivel terciario", no obstante, para comprender "la dimensión y las consecuencias de quienes estudian en el exterior, es necesario combinar la información de los países que reciben a dichos estudiantes mediante recolecciones internacionales de datos». En este aspecto, la Encuesta de Educación de UNESCO facilita datos de estudiantes internacionales provenientes de «118 países o territorios, lo que representa el 77\% de la matrícula total de nivel terciario en el mundo», lo cual ofrece «una posibilidad excepcional para examinar la movilidad estudiantil desde la perspectiva del país de origen y del país donde se realizan los estudios, mediante un seguimiento de las matrículas alrededor del mundo» (UNESCO, 2006: 35).

El UIS informa sobre los principales «hallazgos» por regiones, según la tasa de movilidad hacia el extranjero, la cual expresa «el número de estudiantes internacionales de una región o país como porcentaje de todos los estudiantes de nivel terciario en esa región o país» y también "cuán probable es que los estudiantes continúen su educación en el exterior». Según el UIS, existen varias razones para salir de su propio país a estudiar en el exterior: «la experiencia de estudiar y de vivir en el extranjero, como preparación para vivir en un mundo cada vez más globalizado, la falta de acceso a la educación terciaria en sus países de origen, la oportunidad de una educación de mejor calidad que la ofrecida en sus países de origen» (UNESCO, 2006:37).

Así pues, «una alta tasa de movilidad hacia el exterior puede indicar un gran interés por hacer estudios en el extranjero o también un déficit en la prestación de servicios educativos en el país». Por ejemplo, África Subsahariana tiene «la más alta tasa de movilidad hacia el extranjero (5,9\%), lo que representa casi tres veces más que el promedio mundial». Esta estadística quiere decir que «uno de cada 16 estudiantes de esa región estudia en el exterior» y que, en general, «los países con mucha población joven tienen los números absolutos de estudiantes internacionales más elevados y la mayores proporciones de estos estudiantes por país de origen» (UNESCO, 2006:37).

En el contexto europeo, el Espacio Europeo de Educación Superior (EEES), impulsado por el proceso promovido desde la Declaración de Bolonia en 1999, ha supuesto una plataforma de atracción para estudiantes provenientes de cualquier lugar del mundo. La misma Declaración de Bolonia contiene una serie de intenciones en torno al interés de aumentar la competitividad y atraer talento internacional (Capilla, 2016) a través de ayudas al estudio y acciones enfocadas al incentivo de la movilidad (Valle y Garrido, 2009).

El presente trabajo se desarrolla dentro de los estudios realizados sobre la movilidad internacional de estudiantes en la educación superior internacional (Altbach y Knight, 2006; Luchilo, 2006; Altbach, 2008; Haug, 2010; Gürüz, 2011; Costa y Faria, 2012; Bermúdez, 2015; Parra, 2016) que, multidimensionalmente, abordan las dinámicas y las tendencias relati- 
vas a los flujos migratorios de estudiantes a nivel local y global. Los diversos enfoques de abordaje académico y su interpretación van desde el cálculo de la competitividad en la enseñanza superior (Bermúdez, 2015) a la economía global del conocimiento (Gürüz, 2011) o al estudio de las desigualdades generadas por la neoliberalización de las universidades (Zambrana y Manzano, 2004).

En este sentido, considerando la inclusión del aspecto intercultural y epistemológico originado por la propia movilidad de estudiantes, el estudio se complementa con el concepto de ecosistema de saberes, fundamentado en la ecología de saberes de Boaventura de Sousa Santos (2010), y definido como un conjunto de conocimientos científicos y no científicos que se interrelacionan entre sí a través de unos procedimientos de inputs-outputs (entradas y salidas) de aprendizajes compartidos en un lugar determinado. Según Santos, en la Universidad del siglo xxi, la ecología de saberes se presenta como

la promoción de diálogos de saber científico y humanístico que la universidad produce y los saberes legos, populares, tradicionales, urbanos, campesinos, provincianos, de culturas no occidentales (indígenas de origen africano, oriental, etc.) que circulan en la sociedad. [ ] Comienza a ser socialmente perceptible que la universidad, al especializarse en el conocimiento científico y al considerarlo la única forma de conocimiento válido, contribuyó activamente a la descalificación e inclusive a la destrucción de mucho conocimiento no científico y con eso, contribuyó a la marginalización de los grupos sociales que solamente disponían de esas formas de conocimiento (Santos, 2007: 67).

Asimismo, se está produciendo una desigualdad creciente entre el saber científico y el saber popular, dándose la situación de que «la posesión del saber, los productos del saber, la infraestructura de tecnologías de la información y similares están casi exclusivamente en manos de instituciones, corporaciones y grupos de intereses localizados en el Norte» (Altbach et al., 2006: 15). La conquista de estos saberes científicos «altamente valorados por la sociedad global» supone el «acceso a la elite», aunque las posibilidades de conseguirlo sean mínimas para los «hijos de campesinos, obreros, empleados o pequeños comerciantes» y lleve implícita una aculturación (Bourdieu y Passeron, 2009: 39).

Para Bauman (2002), cualquier sistema social y cultural intenta mantener un equilibrio y trata de preservar su identidad. En caso de realizarse algún tipo de movimiento hacia el interior de sus límites y fronteras, el propio sistema establece «las reglas de admisión y evalúa los resultados de adaptación» (Bauman, 2002: 34-35). Esto produce una asimilación de esos elementos que intentan introducirse en el sistema y que tienen que transitar por «una calle de un solo sentido» (Bauman, 2002: 34-35). En este sentido de unidireccionalidad, Altbach (2006) y Zúñiga (2009) manifiestan que las movilidad internacional de estudiantes está construida desde los países occidentales del Norte, especialmente, y «del mismo modo que la globalización ha tendido a hacer que se concentren la riqueza, el saber y el poder en manos de quienes ya los poseían, la movilidad académica inter- 
nacional ha favorecido a sistemas e instituciones educativos ya desarrollados» (Altbach et al., 2006: 15). Esto ha beneficiado la desigualdad de la educación superior internacional, ya que las «iniciativas suelen ir en el sentido Norte-Sur» (Ibídem) y provoca que el ritmo de las movilidades esté fiscalizado y dirigido por los países occidentales (Altbach et al., 2006; Zúñiga, 2009), ya que, aunque «la internacionalización es mucho más que una calle de un solo sentido y no se puede negar que llena vacíos importantes en el tercer mundo, está ampliamente controlada por el Norte» (Altbach et al., 2006: 15). Efectivamente, los datos de la UNESCO (2009) confirman que la unidireccionalidad es patente en la globalidad de la movilidad internacional de estudiantes. La gran masa estudiantil que emigra o se desplaza en sentido Norte-Sur desde las periferias a países desarrollados y con un sistema de universidades altamente competitivo a nivel mundial (Wallerstein, 2007), especialmente Estados Unidos, es claramente superior al sentido contrario SurNorte. Las movilidades de estudiantes universitarios están cada vez más estructuradas en base a desplazamientos desde países periféricos y subalternos a un centro epistemológico occidental, basado en el saber científico, hegemónico y, en este caso, eurocéntrico (Wallerstein, 2001 y 2007).

Por otro lado, el modelo sistémico se aplica a este concepto de movilidad para referirse a la situación que aparece en un país o ámbito académico en cuanto a las relaciones propiciadas entre saberes, conocimientos y culturas diferentes. Este modelo es de carácter dinámico e híbrido (Bhabha, 2002), teniendo en cuenta, no obstante, que esa hibridez está muy mediatizada por la hegemonía del saber científico que habita mayoritariamente en las universidades europeas y occidentales y que, a su vez, es reticente a dialogar horizontalmente con los saberes tradicionales. Las movilidades de estudiantes producen una acumulación mayor o menor de estos saberes que transgreden también los escenarios académicos y conviven en los países de acogida como otra forma de inmigración. Estos ecosistemas de saberes registran unos inputs de estudiantes provenientes de otras regiones mundiales que producen unas morfologías diferenciadas en los países de destino, producto de la suma de las razones aducidas anteriormente por el UIS (UNESCO, 2006: 37), y añadiéndose a éstas la razón motivada por la afinidad cultural, lingüística e histórica.

En este sentido, algunos estudios previos (Caoili y Valenzuela, 2000; Abrahamsen, 2003; Luchilo, 2006; Motivans, 2009; Francis y Lydia, 2016) argumentan que la relación poscolonial de las antiguas colonias con la exmetrópoli es una de las razones fundamentales por las cuales se llevan a cabo la movilidad de estudiantes. Las ayudas al estudio fomentaron una cooperación entre países que provocaron un aumento de las movilidades y las relaciones académicas e interinstitucionales ya desde finales del siglo xIx, especialmente destinada a las élites económicas y sociales. En el caso africano, Francis y Lidia afirman que

African students tend to migrate to countries formerly included as colonies of the same European power as their own, largely because of derived cultural capital, language facility, and a somewhat shared history. Thus, post-colonial associations are a significant influence on international student mobility (Francis \& Lydia, 2016: 183). 
Así pues, existe cierta lógica colonial para elegir como país de destino o acogida a un país huésped que tenga unas similitudes lingüísticas o culturales con el país de origen y que no presenten tantas dificultades de adaptación y subsistencia. Como expone Zúñiga en un estudio cuantitativo realizado a estudiantes mejicanos y mejicanas sobre movilidad internacional, «las principales dificultades que enfrentaron los estudiantes son las concernientes a la integración al sistema universitario extranjero, el cual presenta diferencias significativas con el sistema educativo de la institución de origen, así como las vinculadas al bajo dominio del idioma y al desconocimiento de la cultura extranjera» (Zúñiga, 2009: 133) y que, por razones históricas derivadas del periodo colonial, se hayan creado unos nexos culturales, lingüísticos y de inter-dependencia económica, política o simbólica que condicionan estos intercambios de movilidad internacional.

\section{METOdOLOGÍA}

El estudio se ha llevado a cabo mediante la realización de un tratamiento estadístico y un análisis cuantitativo de las bases de datos publicadas por UNESCO a través de su Institute for Statistics (UIS). Utilizando esta fuente, se ha recopilado la información sobre la movilidad internacional de estudiantes para su examen a través de Excel, elaborando nueva información estadística y produciendo índices, además de tablas y gráficos para la comprensión visual de la morfología de cada ecosistema de saberes. Así mismo, la información del UIS ha sido contrastada con los datos que aporta Eurostat sobre esta temática, no variando las cifras significativamente. Los datos de estas movilidades no son datos anuales, sino que se han acumulado por país de origen y destino durante el periodo comprendido entre los años 2000 y 2013, produciendo una nueva matriz única de movilidad internacional, en la cual, se puede observar cuánta movilidad total ha habido desde el país de origen al de destino durante este principio del siglo xxI.

Una vez conocidas las cantidades y el origen de las movilidades internacionales hacia estos países del arco atlántico europeo, se han superpuesto al mapa de la colonización histórica, es decir, se ha relacionado la afinidad histórico-colonial de cada país que realiza esa movilidad con el país anfitrión o de destino. El resultado de esta superposición es una nueva matriz que asocia las variables de movilidad y colonialismo.

Por otro lado, para medir los flujos de movilidad hacia estos países según la región mundial de origen y poderlos comparar, ha sido calculado el Índice de Elegibilidad de cada país de destino aplicando la metodología propuesta por Valle y Garrido (2009), utilizada para las movilidades ERASMUS intraeuropeas. Para ello, en primer lugar, han sido calculados los Coeficientes de Elección de cada país de destino, basándonos en la decisión de cada estudiante de elegir un país u otro como destino para realizar la movilidad. Posteriormente, se calcula el valor resultante de la suma de cada uno de estos coeficientes de cada país de destino, resultando el Índice de Elegibilidad, el cual está comprendido entre 0 y 1 . Un país cuyo valor acumulado sea 0 , significa que ninguna región mundial lo elige como destino. 
Por el contrario, en el caso de obtener un país el valor 1, quiere decir que es el más elegido por las regiones para realizar la movilidad. Los valores intermedios suponen un gradiente entre estos valores extremos. Así mismo, Valle y Garrido (2009: 112) establecen una escala nominal y cualitativa para los resultados obtenidos:

\begin{tabular}{|c|l|}
\hline Índice de elegibilidad & \multicolumn{1}{|c|}{ Valoración } \\
\hline $0-0,19$ & Nula \\
\hline $0,20-0,39$ & Escasa \\
\hline $0,40-0,59$ & Moderada \\
\hline $0,60-0,79$ & Alta \\
\hline $0,80-1$ & Muy alta \\
\hline
\end{tabular}

\section{ANÁLISIS DE LAS MOVILIDADES HACIA REINO UNIDO, PORTUGAL, FRANCIA Y ESPAÑA}

Los países europeos seleccionados para analizar la movilidad de estudiantes internacionales a Europa describen, en general, las pautas que se establecen en el resto de movilidad mundial señalado por la UNESCO (2006:34), con un incremento en la primera década del siglo xxi (Figura 1), más concretamente, en el periodo comprendido entre el año 2000

Figura 1. Incrementos de movilidad y movilidad total por regiones mundiales (periodo 2000-2013)

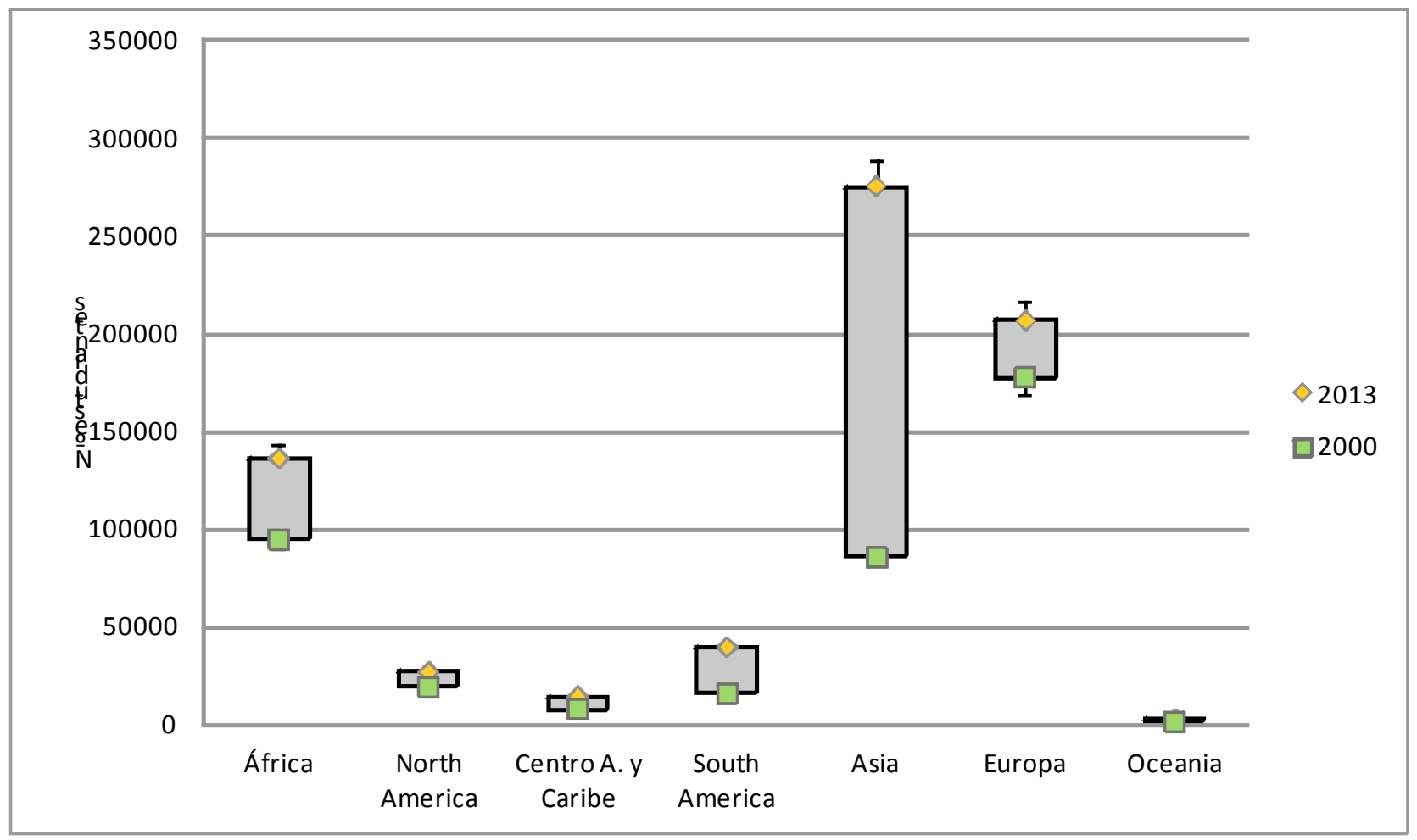

Fuente: elaboración propia. Datos UNESCO-UIS. 
y 2013. Esta movilidad aumenta desde todas las regiones mundiales y es de destacar el crecimiento de la movilidad asiática, especialmente China e India que se desplazan mayoritariamente a Reino Unido, con un diferencial a favor de 189.625 estudiantes para ese continente, lo que supone el 63,4\% del incremento de la movilidad internacional que se desplaza a estos cuatro países europeos para realizar sus estudios universitarios y ocupa, en 2013, el primer puesto en el cómputo total con más de 275.000 estudiantes que conviven con los estudiantes autóctonos en estos ecosistemas de saberes europeos.

En un lugar más secundario y por orden descendente del incremento de la movilidad, se encuentran las regiones de África (13,8\%), Europa (9,8\%) y América del Sur (7,9\%). En el caso de África es especialmente destacable el incremento de Nigeria, así como de Senegal, Argelia, Túnez, Marruecos y Guinea, los cuales se desplazan mayoritariamente a Reino Unido y Francia. El tercer incremento más importante, en términos cuantitativos, corresponde a Europa que, a pesar de encontrarse a una distancia más cercana que Asia, ocupa un segundo puesto en cuanto a la movilidad total de sus estudiantes con más de 200.000 en el año 2013. El cuarto puesto, tanto en incremento entre 2000 y 2013 como de posición total de movilidades, lo ostenta América del Sur, destacando Brasil, Colombia y Perú, por este orden, y se desplazan a España, Francia y Portugal, principalmente. El resto de regiones, aunque han aumentado sus desplazamientos, sufren un incremento menor de la movilidad en el periodo correspondiente.

En cuanto a las movilidades que se producen según las diferentes regiones mundiales a cada país (Figura 2), es especialmente llamativo la destacada cantidad de movilidad que se producen en Reino Unido, principalmente de Asia y el resto de países de Europa. El caso de China supone una inflación de la movilidad a nivel mundial (Yu \& Jin, 2014), un ejemplo de la estrategia del gigante asiático por mantener su creciente importancia en el escenario económico mundial con la mayoritaria presencia de la juventud china en las universidades con mayor ranking mundial, en el denominado "golden triangule» de las universidades de Oxford, Cambridge y Londres (Khattab \& Fenton, 2014).

Al igual que ocurre con el estudiantado chino, las y los estudiantes europeos optan mayoritariamente por desplazarse al centro del conocimiento científico y hegemónico de la epistemología dominante occidental (Santos, 2010). Asia y Europa, al igual que América del Norte, eligen el atractivo de la órbita universitaria anglosajona, mientras que la mayor parte del estudiantado africano se decide por desplazarse a Francia, especialmente, las provenientes de las antiguas colonias del norte de África. Así mismo, España y Portugal confirman su condición de potencias satélites del conocimiento y se sitúan en la periferia de las preferencias de movilidad de los estudiantes internacionales. Únicamente se ratifica la hegemonía de España como lugar de destino de la mayor parte de las movilidades originarias del territorio meridional americano. 
Figura 2. Distribución de la movilidad por regiones mundiales (periodo 2000-2013)

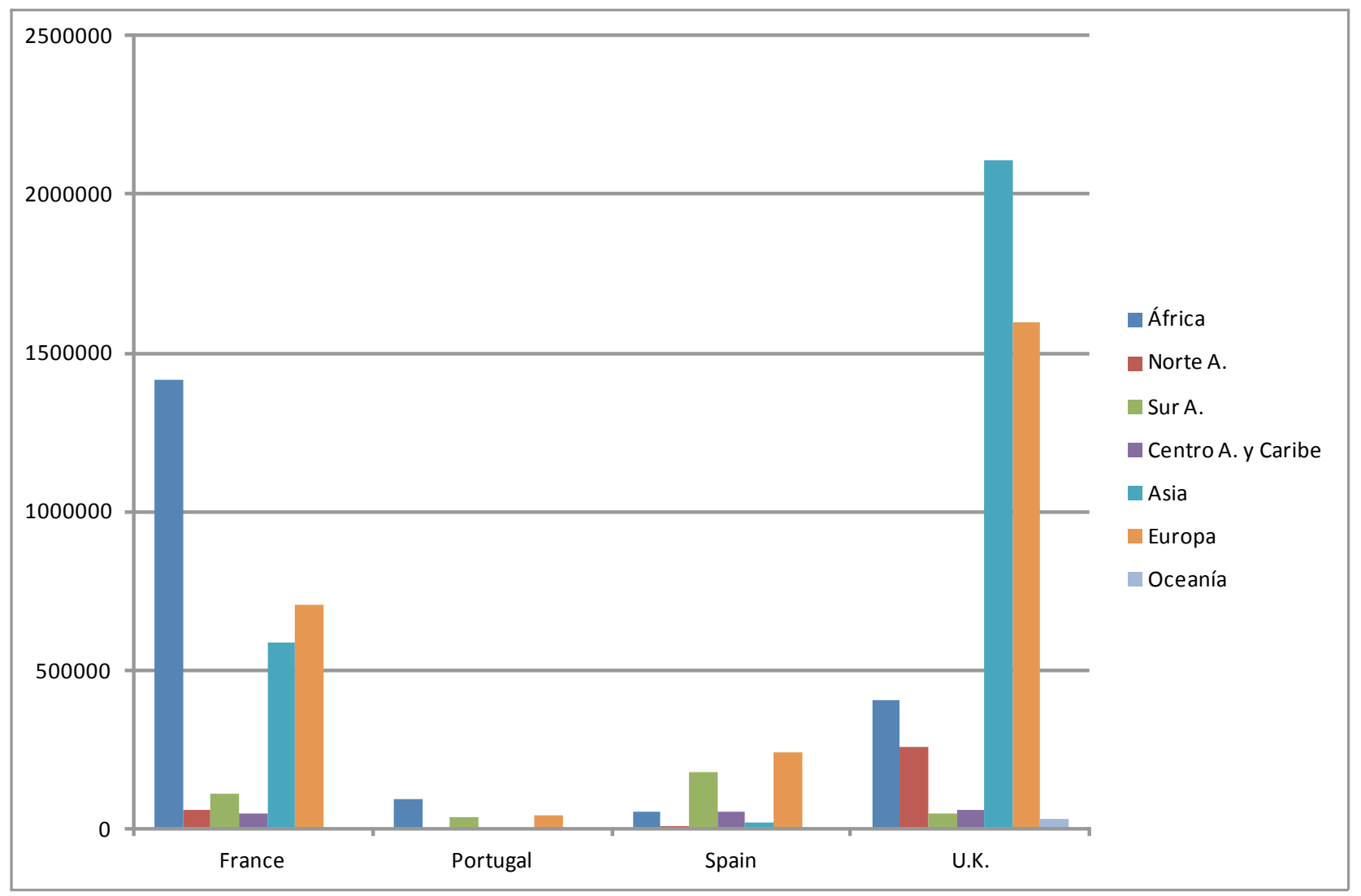

Fuente: elaboración propia. Datos UNESCO-UIS.

El análisis de los datos refleja la distribución de las movilidades (inputs) en cada uno de los ecosistemas de saberes y confiere a cada uno de los países anfitriones una morfología diferenciada (Figura 3). En el caso de Portugal, la movilidad de estudiantes está caracterizada por la aportación sustancial de movilidad cultural lusófona y postcolonial, representando el $66,5 \%$ de las movilidades. El caso de Francia posee una morfología similar, ya que los países que más movilidad aportan son los que se encuentran dentro de una órbita cultural francófona y de relación postcolonial con la exmetrópoli, como son los casos de Marruecos, Argelia, Túnez, Senegal y Camerún con el 33,3\% de la movilidad.

Los casos de España y Reino Unido son ligeramente diferentes. Por un lado, en Reino Unido ocurre que la movilidad china de estudiantes es muy amplia (como también se aprecia en Francia), absorbiendo así parte de la hegemonía de este país asiático a nivel global. No obstante, existe una movilidad relacionada con los ecosistemas de saberes anteriores, basada en la afinidad lingüístico-cultural e histórica, como se aprecia en la relevancia de movilidades provenientes de India, Malasia, Nigeria, Hong Kong o Chipre. El caso de España, por otro lado, es más heterogéneo y su movilidad contiene tanto de origen cultural (Colombia, México, Perú, etc.) como de países europeos preferentemente. 
Figura 3. Comparativa de morfologías de los ecosistemas de saberes (2000-2013)

\section{Portugal}

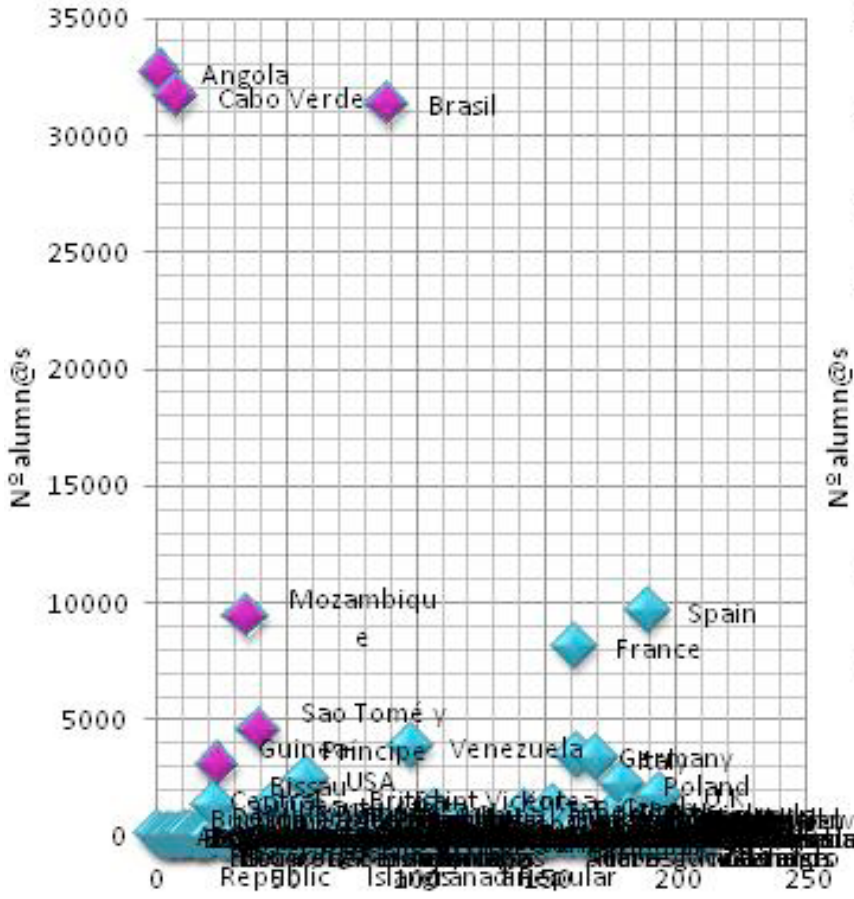

España

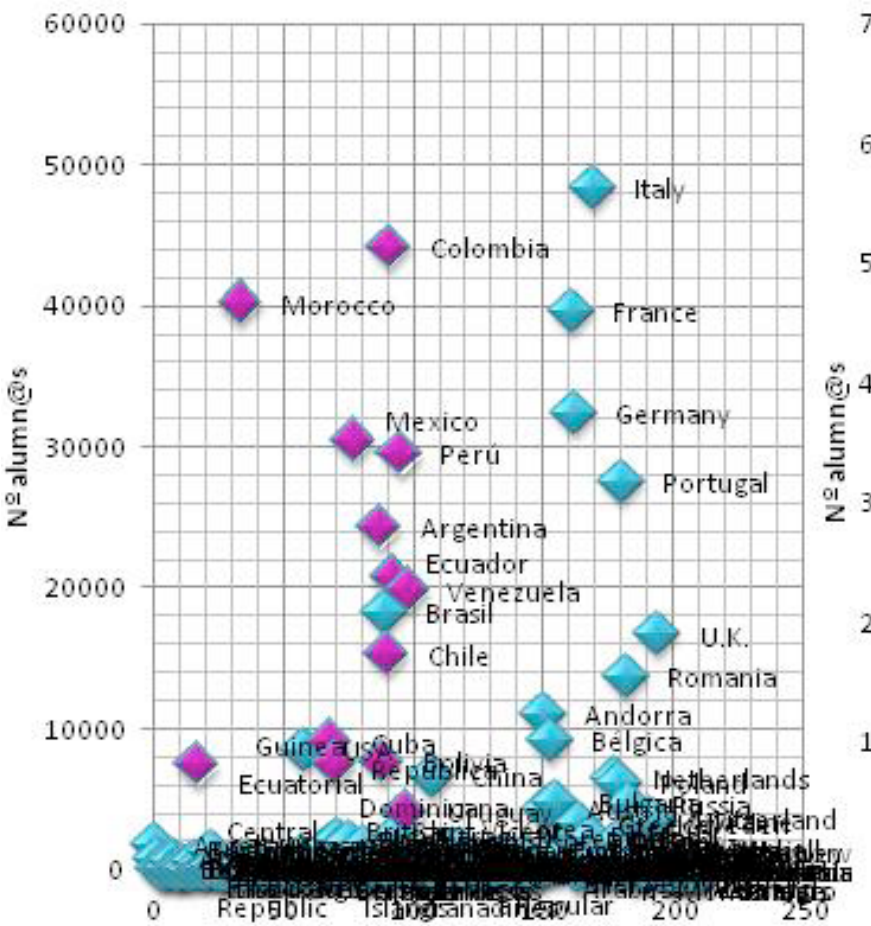

Francia

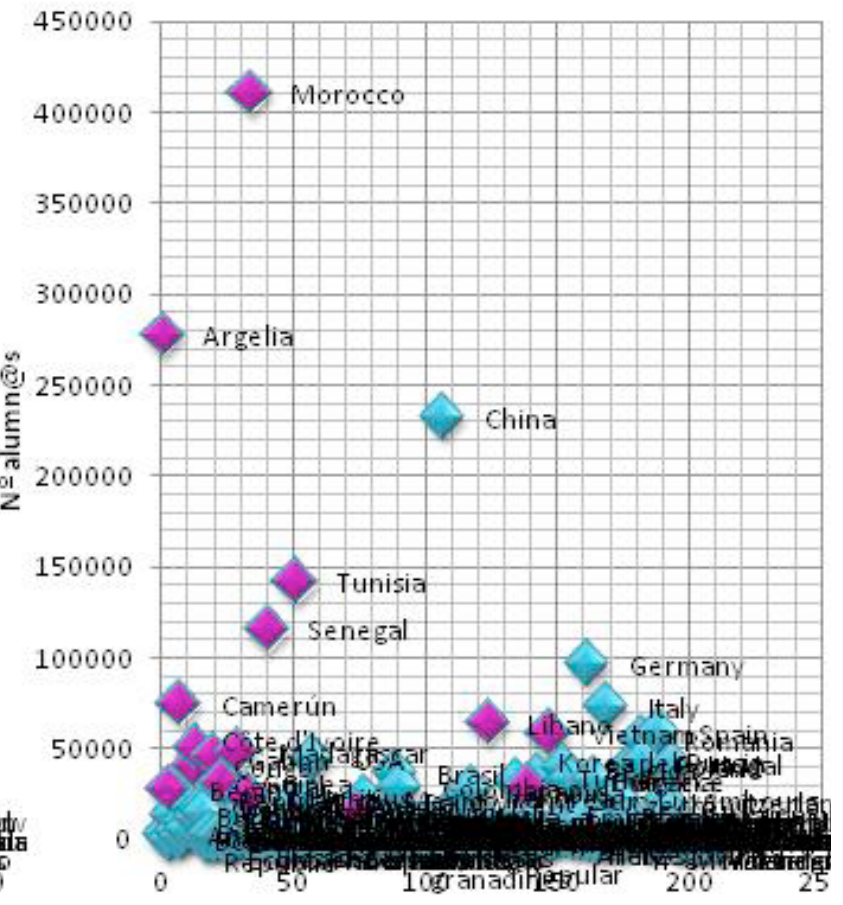

U.K.

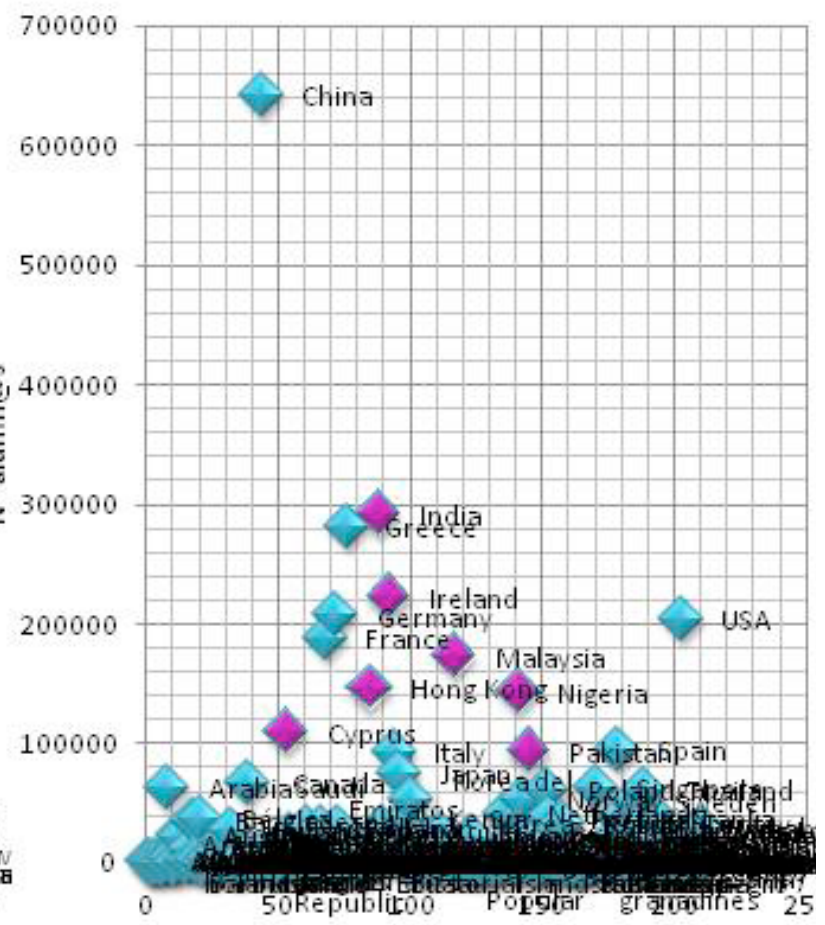

- PAISES COLONIZADOS

- PAISES NO COLONIZADOS

Fuente: elaboración propia. Datos UNESCO-UIS. 
Esta correlación cultural está basada, principalmente, en el proceso de colonización que, durante siglos, desarrollaron los países europeos seleccionados en todos los continentes. El reparto de los territorios colonizados supuso la implantación de los modos de vida, costumbres culturales e idiomas occidentales en la poblaciones locales a través de un proceso de «blanquización» (Fanon, 1973; Freire, 2004) y colonización de las mentes (Ngũgĩ, 1986) que originó una aculturación y una dependencia de las metrópolis, también en el ámbito educativo (Galeano, 1999; Freire, 2004). La movilidad de estudiantes desde las antiguas colonias a universidades europeas con el fin de formar y europeizar a las poblaciones no civilizadas viene dándose desde hace siglos, no es un fenómeno nuevo, aunque era menos masificado que en la actualidad (Wiredu, 2011). Estas movilidades estaban destinadas a estudiantes elegidos provenientes de las colonias de ultramar que conocían el idioma del colonizador y habían sido "asimilados» (Memmi, 1974). Actualmente, el destino de la movilidad todavía guarda relación con aquel idioma del colonizador, como se observará a continuación.

A través de la intersección de los datos de movilidad de la UNESCO con el mapa lingüístico de los idiomas oficiales en cada uno de los países mundiales, se confiere una nueva información en la que se aprecian las preferencias de las alumnas y alumnos internacionales en su movilidad a Europa (Figura 4). La superposición de las dos variables confirma la ten-

Figura 4. Igualdad entre el idioma hablado en el país de origen y en el país anfitrión

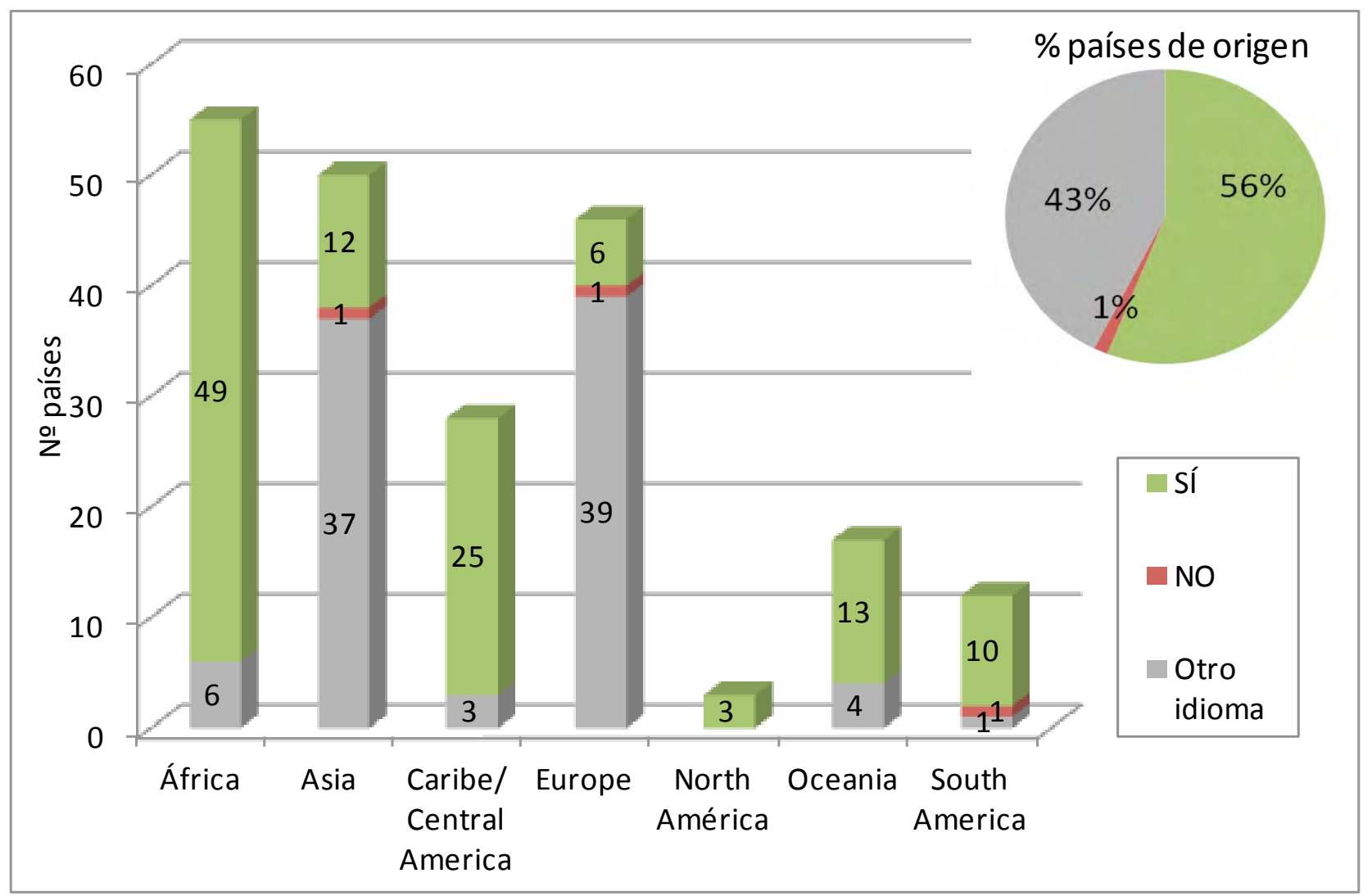

Fuente: elaboración propia. 
dencia a elegir un país de destino que guarde una asociación entre idioma-cultura y movilidad. Los flujos de estudiantes que se desplazan a otros países son la continuidad de la dinámica de las movilidades universitarias y educativas que se vienen desarrollando desde mediados del siglo xx, como, por ejemplo, en Portugal (Gusmão, 2005; Costa \& Faria, 2012), cuya escasa movilidad proveniente del exterior está compuesta, en su mayoría, por estudiantes provenientes de los países excoloniales africanos.

La relación entre el idioma que habla el estudiante en su lugar de origen con el idioma que se habla en el lugar de destino está claramente asociada en África, América del Sur y Central, Norte América y Oceanía. Por el contrario, no se da la misma situación en Asia y Europa. En África, América Central y América del Sur, aproximadamente 9 de cada 10 países que deciden realizar la movilidad internacional guardan una relación lingüística directa con el país de destino. En el caso de Oceanía, son 8 de cada 10. Los norteamericanos llegan a ser el $100 \%$ de estudiantes, mientras que, en el sentido opuesto, la relación asiática se mantiene en porcentajes mucho más bajos debido, en gran medida, a la diversidad lingüística que existe en esas áreas mundiales (que también engloba a países de lengua árabe), las cuales han mantenido su lengua nativa como idioma oficial, no la lengua de la antigua metrópoli. En este sentido, las movilidades de los países asiáticos se reducen a aproximadamente 3 de cada 10. Europa, con una mayor diversidad lingüística oficial, presenta una relación movilidad-idioma de 1 de cada 10 países.

En cómputos globales, la igual relación del idioma del estudiante con el idioma del país de destino está representada en el 56\% de los países, mientras que la no relación se sitúa en el 1\%. El 43\% restante de los países que realizan la movilidad a Europa posee un idioma diferente. Eso quiere decir que los estudiantes de los países de lengua oficial española, por ejemplo, localizados fundamentalmente en América Latina van a desplazarse a España de manera mayoritaria en el $100 \%$ de los casos. En los países con lengua oficial portuguesa, salvo Macao que mantiene una fuerte relación con Reino Unido, lo hará a Portugal. Así mismo, los de lengua oficial francesa, salvo Suiza que también se desplaza principalmente a Reino Unido, lo hará a Francia, especialmente desde África y Asia, y los de lengua oficial inglesa se desplazarán mayoritariamente a Reino Unido en el 100\% de los casos, sobre todo desde Asia, África, América del Norte y Oceanía (como se aprecia en la Figura 2). Hay que señalar, así mismo, que tanto Reino Unido como Francia todavía poseen un gran número de colonias adscritas a su administración y gobierno, las cuales mantienen un grado de dependencia en la educación superior, entre otros aspectos, y se ve reflejado en el alto Coeficiente de Elección por estos países europeos. Por otro lado, estos altos Coeficientes de Elección de las colonias y excolonias producirán, a su vez, un aumento del Índice de Elegibilidad, entendido como «el potencial de atracción de unos países sobre otros a tenor de los flujos de estudiantes» (Valle et al., 2009).

En este sentido, el Índice de Elegibilidad (IE) presenta unas diferencias sustanciales entre cada país (Figura 5). Portugal sostiene unos niveles muy bajos de elegibilidad, con un $I E$ de 0,05, estableciendo una valoración Nula. Un poco mejor situado estaría España, con 
Figura 5. Índice de Elegibilidad y proporción de movilidad (2000-2013)

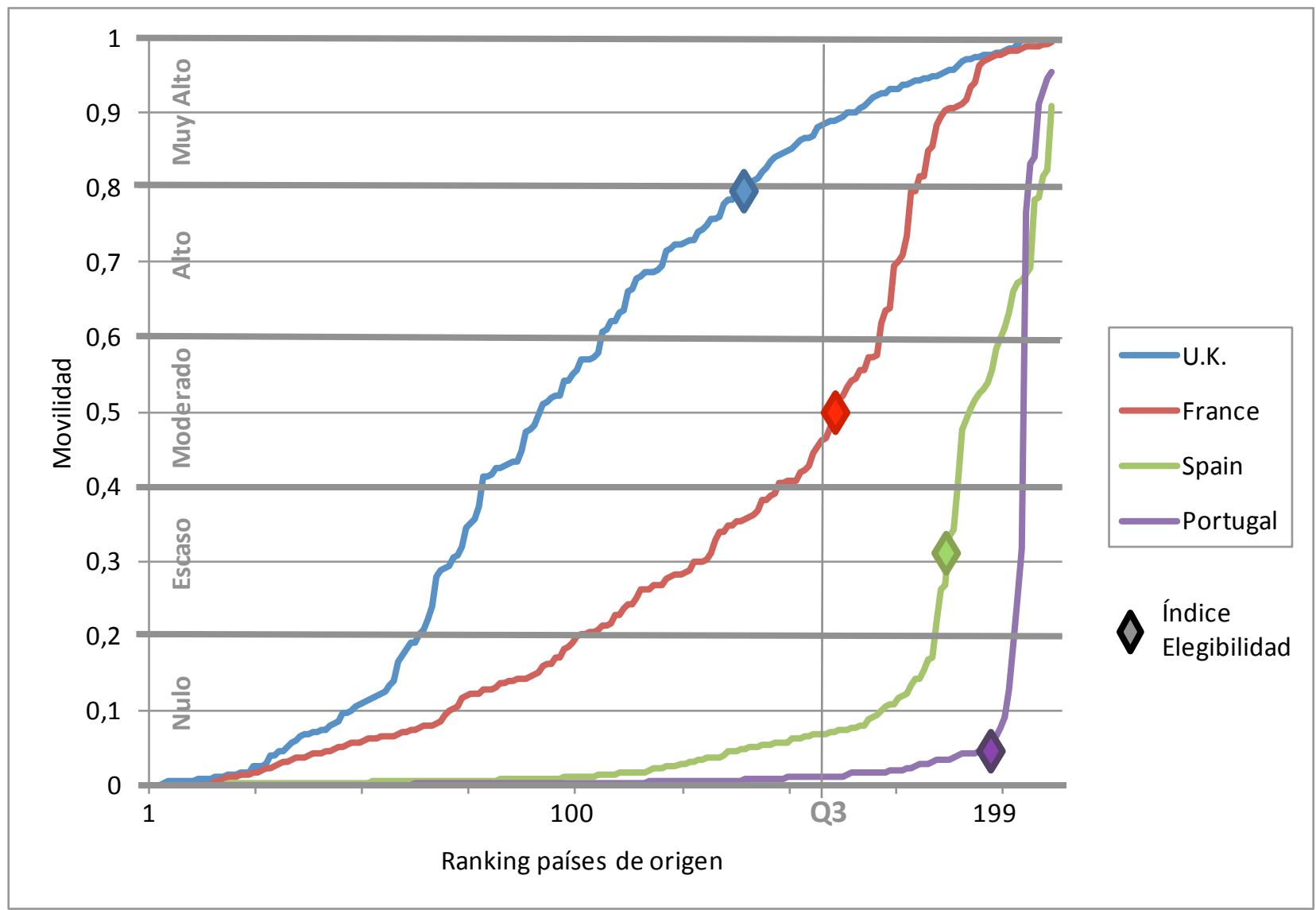

Fuente: elaboración propia.

un 0,31, que, aún así, obtiene una valoración Escasa de elegibilidad. El aprobado lo consigue Francia, con un 0,5, y una valoración Moderada. Y finalmente, en primer lugar, se sitúa el Reino Unido que obtiene un $I E$ de 0,79 y una valoración de elegibilidad Alta o, prácticamente, Muy Alta.

En el gráfico, se puede observar también la proporción de estudiantes que eligen realizar la movilidad a cada país de destino, tomando en cuenta todos los países de origen y ordenados de menor a mayor proporción. En dicha relación es significativo que, a partir del Q3, Reino Unido es elegido por más del 90\% de los estudiantes de esos países, Francia no llega al 50\%, España se encuentra por debajo del 10\% y Portugal prácticamente situado a niveles inexistentes de elegibilidad. Por lo tanto, es evidente el desequilibrio ocasionado entre los países de destino, seleccionados como países anfitriones de la movilidad internacional de estudiantes.

No obstante, hay que tener en cuenta que el estudio está realizado a nivel global, es decir, considerando a todos los países del mundo que realizan la movilidad a estos países de destino seleccionados y que han sido tratados con los datos aportados por el Instituto de Estadística de la UNESCO desde el año 2000 al 2013. Por lo tanto, la comparativa puede variar si se añadiese un país de destino con un mayor número de movilidad que el resto de paí- 
ses. Es decir, en el ejemplo de la selección de estos cuatro países, si hubiera un país en Europa con una mayor movilidad que Reino Unido y se incluyera en la comparativa, cambiarían los resultados actuales. Lo cual es improbable ya que, al menos de momento, el Reino Unido sólo se ve superado por Estados Unidos como país anfitrión en el ranking mundial de movilidad internacional de estudiantes. Así mismo, se ha analizado que, a lo largo de la primera década del siglo xxi, los IEs de los cuatro países europeos no han tenido mucha variación y han oscilado en torno a los valores medios calculados.

\section{CONCLUSIONES}

A través de este artículo, se ha descrito y comparado la movilidad internacional de estudiantes que llevan a cabo todos los países y territorios del mundo hacia los países europeos seleccionados. Portugal, Francia, Reino Unido y España son países receptores de estas movilidades y, tal como describe la UNESCO (2009), aumentan las continuas remesas de estudiantes extranjeros que se desplazan a realizar estudios superiores a los centros universitarios europeos. A nivel global, estos desplazamientos se caracterizan por la unidireccionalidad, es decir, los países que movilizan masivamente a sus estudiantes no son países receptores y están localizados fuera de la centralidad del conocimiento científico y del saber hegemónico (Santos, 2007).

Los cuatro países seleccionados presentan unas morfologías análogas en sus ecosistemas de saberes. Los aportes o inputs de estudiantes al ecosistema de acogida están identificados con la procedencia de estudiantes externos desde países que mantienen una relación lingüística, cultural e histórica muy ligada a la herencia colonial. La asociación directa entre el idioma de origen y el idioma de destino en el país donde se lleva a cabo la movilidad es positiva en la mayor parte de los casos y sólo Macao, Suiza y Brasil, en cómputos globales, son la excepción a esta norma. No obstante, tanto Portugal como Francia muestran una mayor presencia de estudiantes provenientes de sus (ex)colonias, sobre todo africanas, lo cual confiere una imagen de la africanización de su movilidad internacional y, sobre todo, del desequilibrio producido en los desplazamientos entre países (ex)colonizados y no colonizados.

Por otro lado, los desplazamientos de estudiantes que provienen de colonias actuales de «ultramar», especialmente con ciudadanía inglesa y francesa, forman parte de esta movilidad internacional y no se han considerado movilidades internas propias de cada país. En los casos de Francia y Reino Unido, estas colonias son especialmente numerosas y esto, además de negar las identidades culturales intrínsecas de esos territorios no europeos, conllevaría una contrariedad ética al secundar una continuidad del régimen colonial que contraviene la Carta Magna de la Descolonización declarada por la ONU en 1960. Esta distinción, no obstante, posibilita que los Índices de Elegibilidad de estos dos países puedan ser más altos, respecto de los otros dos: España y Portugal, ya que, al tener un mayor número de colonias, aumentan los Coeficientes de Elección a su favor. 
En este sentido, el cálculo del Índice de Elegibilidad ha permitido comparar cuantitativamente las movilidades de los cuatro países observados, así como su clasificación cualitativa de elegibilidad. Reino Unido y Francia se ubican en posiciones altas o moderadas de elegibilidad de la movilidad internacional y, por el contrario, España y Portugal se localizan en puestos de elegibilidad escasos o nulos.

La elaboración del Índice de Elegibilidad y el análisis de la relación entre idioma-movilidad han servido para construir varios escenarios de movilidad complementarios. Por un lado, en términos cuantitativos de IE, diferencia a unos países de destino situados en una relación Centro-Periferia, donde Francia y, especialmente, Reino Unido absorben una gran parte de la movilidad internacional de estudiantes con IEs medios y altos, mientras que España y Portugal ostentan valores muy bajos. Por otro lado, en términos cualitativos basados en el país de origen de la movilidad, distingue dos ecosistemas de saberes diferenciados correspondientes a una Movilidad Doméstica, de «andar por casa», moderna, dicotómica y colonial, representada por Francia y Portugal; y una Movilidad Globalizada, líquida, múltiple y posmoderna, más presente en Reino Unido y España. Estos cuatro escenarios se solaparían de la siguiente manera:

\begin{tabular}{|l|l|l|}
\hline Movilidad & Globalizada & Doméstica \\
\hline Centro & U.K. & Francia \\
\hline Periferia & España & Portugal \\
\hline
\end{tabular}

\section{BIBLIOGRAFÍA}

Abrahamsen, R. (2003). African studies and the postcolonian challenge. African affairs, 102, pp. 189-210.

Altbach, P.G. y Knight, J. (2006). Visión panorámica de la internacionalización en la educación superior: motivaciones y realidades. Perfiles educativos, vol. XXVIII, 112, pp. 13-39.

Altbach, P.G. (2008). Funciones complejas de las universidades en la era de la globalización. In: Global University Network for Innovation, Educación superior en el mundo 3. Madrid: Ediciones Mundi-Prensa, pp. 5-14.

Bauman, Z. (2002). La cultura como praxis. Barcelona: Paidós.

Bermúdez, R.E. (2015). La movilidad internacional por razones de estudio: Geografía de un fenómeno global. Migraciones internacionales, 8(1), pp. 95-125.

Bhabha, H.K. (2002). El lugar de la cultura. Buenos Aires: Manantial.

Bourdieu, P. \& Passeron, J.C. (2009). Los herederos: los estudiantes y la cultura. Buenos Aires: Siglo XXI. 
Caoili, O., \& Valenzuela, E.A. (2000). International of higher education: Patterns, trends, status and directions. In: M.D. Valisno (Ed.), The reform and development of higher education in the Philippines. Manila: UNESCO Philippines, pp. 153-186.

Capilla, A. (2016). La estrategia para la internacionalización de las universidades españolas. In: M. Martín (Coord.), Internacionalización de la educación superior en Iberoamérica: miradas y perspectivas. Madrid: Universidad de Alcalá de Henares, pp. 37-52.

Costa, A.B. y Faria, M.L. (2012). Formação superior e desenvolvimento: estudantes universitarios africanos em Portugal. Coimbra: Almedina.

Fanon, F. (1973). Piel negra, máscaras blancas. Buenos Aires: Abraxas.

Francis, N., \& Lydia, E. (2016). Student Mobility in International Education: The Case in Ghana. International Journal of Academic Research in Business and Social Sciences, 6(6), pp. 177-188.

Freire, P. (2004). Cartas a Guinea-Bissau: apuntes de una experiencia pedagógica en proceso Buenos Aires: Siglo XXI.

Galeano, E. (1999). Patas arriba. La escuela del mundo al revés. Madrid: Siglo XXI.

Gürüz, K. (2011). Higher Education and International Student Mobility in the Global Knowledge Economy. New York: State University of New York Press.

Gusmão, N.M. (2005). Os Filhos da África em Portugal. Antropologia, multiculturalidade e educação. Belo Horizonte: Autêntica.

Haug, G. (2010). La internacionalización de la educación superior: más allá de la movilidad europea. La Cuestión Universitaria, 6, pp. 20-29.

Khattab, N. \& Fenton, S. (2014). Globalisation of researcher mobility within the UK Higher Education: explaining the presence of overseas academics in the UK academia. Globalisation, Societies and Education, Vol. 14, Iss. 4.

Luchilo, L. (2006). Movilidad de estudiantes universitarios e internacionalización de la educación superior. Revista CTS, n. ${ }^{0}$ 7, vol. 3, pp. 105-133.

Memmi, A. (1974). Retrato del colonizado precedido por Retrato del colonizador. Madrid: EDICUSA.

Motivans, A. (2009). Tendencias mundiales en materia de movilidad internacional. Boletín EduInfo, Junio-Julio. UNESCO.

Ngũgĩ, W.T. (1986). Decolonising the Mind: the politics of language in african literature. London: Heinemann. 
Parra, M.C. (2016). Movilidad internacional de estudiantes: escenarios de oportunidades para la creación de una clase cosmopolita. Tesis Doctoral, Universidad Pontificia Comillas.

Santos, B.D.S. (2007). La Universidad en el siglo XXI. Para una reforma democrática y emancipatoria de la universidad. La Paz: Plural editores.

Santos, B.D.S. (2010). Descolonizar el saber. Reinventar el poder. Montevideo: Trilce.

Sen, W. (2009). Chinese student migration in Europe. A migration that hobody objects to?. In: H. Kolb y H. Egbert (Ed.), Migrants and markets: perspectives from economics and other social sciences. Amsterdam: Amsterdam University Press, pp.147-167.

UNESCO (2006). Compendio mundial de la educación 2006. Comparación de las estadísticas de educación del mundo. Montreal: Instituto de Estadística de la UNESCO (UIS).

UNESCO (2009). Compendio mundial de la educación 2009. Comparación de las estadísticas de educación del mundo. Montreal: Instituto de Estadística de la UNESCO (UIS).

Valle, J.M. \& Garrido, R. (2009). Movilidad de estudiantes universitarios:¿ Es España atractivo para los estudiantes Erasmus?. Revista Fuentes, 9, pp. 98-117.

Wallerstein, I. (2001). El eurocentrismo y sus avatares: los dilemas de la Ciencia Social. In: W. Mignolo (Comp), Capitalismo y geopolítica del conocimiento: El eurocentrismo y la filosofía de la liberación en el debate intelectual contemporáneo. Buenos Aires: Ediciones del Signo, pp. 97-113.

Wallerstein, I. (2007). Universalismo europeo: el discurso del poder. México: Siglo XXI.

Wiredu, H. (2011). Crítica de Amo a la «Filosofía de la Mente» de Descartes. Africaneando. Revista de actualidad y experiencias. Núm. 07, 3. ${ }^{\circ}$ trimestre, pp. 9-18.

Yu, K. \& Jin, J. (2014). Tuition fees and participation in Chinese Higuer Education: the long march to marketisation anda massification. In: H. Ertl \& C. Dupuy (Ed.), Students, markets and social justices. Higher education fee and student support policies in Western Europe and beyond. Oxford: Symposium Books, pp. 161-182.

Zambrana, L.A. \& Manzano, V. (2004). ¿Hacia dónde camina la Universidad? Reflexiones acerca del EEES. Revista Interuniversitaria de Formación del Profesorado, 18(3), pp. 269-276.

Zúñiga, M. (2009). La movilidad internacional de estudiantes universitarios neoleoneses. Un recuento de las dificultades y las ganancias. Revista Perspectivas Sociales/Social Perspectives, Vol. 11, N. ${ }^{\circ} 1$ y 2, pp. 133-154. 